\title{
Parc National Du Banco, Un Patrimoine Entre Destruction Et Conservation : Realite Et Enjeux D’une Gestion Durable
}

\author{
Akoue Yao Claude \\ Adaman Sinan \\ Université Péléforo Gon Coulibaly de Korhogo \\ Zon Dehenouin Alphonse \\ Université Felix Houphouêt Boigny
}

doi: 10.19044/esj.2016.v13n2p182 URL:http://dx.doi.org/10.19044/esj.2016.v13n2p182

\begin{abstract}
Forest protection and conservation in Ivory Coast and its ressources were always been in political concern. That is why since its independance, Ivory Coast created an important network of protected areas in order to preserve its forest cover and its biodiversity. In spite of these efforts of forest conceptualization, the situation remains in crisis. Indeed the share of the National parks and Reserves undergo deep changes related to the anthropic pressures. Created in 1953 and located in the heart of the economic capital of the Ivory Coast, the National Park value does not escape such a situation. Indeed, the activities undertaken by populations living around this park and the fast urbanisation of the town of Abidjan represent an obstacle to the survival of the park. To suppress this situation, political measurements are installed for better managment of this park. This study aims to analyze the threats related to urban pressure on this park.
\end{abstract}

Keywords: Park, Heritage, Destruction, Conservation, Challenges, Management

\section{Résumé}

La protection et la conservation de la forêt ivoirienne et de ses ressources a été toujours au centre des préoccupations des pouvoirs publics. C'est la raison pour laquelle depuis son accession à l'indépendance, la Côte d'Ivoire a créé un important réseau d'aires protégées dans le but de conserver son couvert forestier et sa biodiversité. Malgré ces efforts de conceptualisation de son couvert forestier, la situation demeure catastrophique. La plus part des Parcs Nationaux et Réserves subissent de profondes mutations liées aux pressions anthropiques. Créé en 1953 et situé 
en plein cœur de la capitale économique de la Côte d’Ivoire, le Parc National du Banco n’échappe pas à une telle situation. En effet, les activités menées par des populations vivant autour du parc et à l'urbanisation rapide de la ville d'Abidjan constituent une entrave pour la survie du parc. Pour juguler cette situation, des mesures et des politiques sont mises en place pour une gestion durable du patrimoine. Cette étude vise à analyser les menaces liées à la pression urbaine qui reste un danger pour la survie du parc

Mots-clés : Parc, patrimoine, destruction, conservation, enjeux, gestion durable

\section{Introduction}

En Côte d'Ivoire, la conservation et la sauvegarde des domaines forestiers permanents de l'Etat ont toujours été au centre des préoccupations des pouvoirs publics. C'est pourquoi, depuis 1960, date d'accession à son indépendance, l'Etat ivoirien conscient de ce fait, a créé un réseau dense d'aires protégées composées de Parcs Nationaux, de Réserves Naturelles et de Réserves botaniques ${ }^{12}$ couvrant près de $16 \%$ de son territoire national(OIPR). D'une superficie totale de 3.474 ha, le Parc National du Banco joue un rôle prépondérant de par sa situation géographique au cœur de la capitale économique ivoirienne. Mais ce parc comme tous les autres subit de très fortes pressions anthropiques (Baligné, 1994). Bordé de quatre communes, ce parc subit les conséquences de l'urbanisation galopante et vertigineuse de la ville d'Abidjan. Cette situation compromet la conservation de cette aire protégée.

Le présent article vise à décrire et à analyser les différentes menaces liées à la pression urbaine notamment les risques de déforestations et de pollutions qui constituent des dangers permanents pour la survie du parc. Autrement dit, il s’agira dans une première partie de décrire les stratégies de conservation du parc. Dans une deuxième partie montrer les impacts des actions anthropiques sur le parc et enfin de présenter les enjeux d'une gestion durable du patrimoine.

\footnotetext{
${ }^{12}$ La différence entre un Parc National et une Réserve Naturelle est : " Un parc est un espace délimité créé par décret et dans lequel on préserve la faune et la flore. On peut y faire des aménagements à n’importe quel moment .Quant à une Réserve naturelle, elle a les mêmes caractéristiques qu'un Parc National mais la différence se situe au niveau de l'aménagement qui est impossible dans une Réserve. Comme exemple lorsqu'un véhicule tombe dans un ravin dans une Réserve il ne peut pas être déplacé mais dans un parc cela est possible ».
} 


\section{Notes methodologiques}

Sur le plan méthodologique, nous avons procédé à une combinaison des approches qualitatives et quantitatives. Ce travail s'appuie sur les données d'enquête bibliographiques (étude documentaire) et d'enquête auprès des gestionnaires du parc (Office Ivoirien des Parcs et Réserves) et des services décentralisés (mairies d’Adjamé, Yopougon, Abobo et d’Attécoubé) qui abritent cette aire protégée. Les populations vivant aux alentours du parc national du Banco ont été également approchées. A cet effet, quatre quartiers ont été ciblés pour l'enquête. Il s’agit de : Sodeci, Abobo-Sagbé, Anonkoua-Kouté, et Andokoi. L’échantillon de la population enquêtée est constitué au total de 110 personnes. Il a été obtenu par la méthode de calcul probabiliste. Cette méthode implique un tirage au hasard. C'est-à-dire qui donne à chaque élément de la population une chance égale d’être choisi. Sa forme stratifiée a porté sur les communautés locales qui présentent une population très hétérogène composée de nombreux sousgroupes. Quant à sa forme simple, elle a été utilisée pour la sélection des agents institutionnels caractérisés par l’homogénéité de leurs composantes.

Tableau1 : Répartition des répondants par quartiers

\begin{tabular}{|c|c|c|}
\hline Quartiers d'enquête & $\begin{array}{c}\text { Nombre de répondants par } \\
\text { zone }\end{array}$ & Pourcentages (\%) \\
\hline ANONKOUA-KOUTE & 28 & 25,45 \\
\hline SODECI & 25 & 22,72 \\
\hline AGBAN-VILLAGE & 05 & 4,54 \\
\hline ABOBO-SABGE & 29 & 26,36 \\
\hline ANDOKOI & 23 & 20,90 \\
\hline TOTAL & 110 & 100 \\
\hline
\end{tabular}

Source : Données d'enquête, Mars-Avril 2016.

En outre, un guide d'entretien a été élaboré pour servir de support aux entretiens avec les responsables de l'aire protégée au nombre de deux personnes et des services décentralisés de l'administration avec trois enquêtés. Un questionnaire a été adressé aux populations vivant dans les villages et quartiers à la périphérie du parc au nombre de 105 personnes.

\section{Strategies de conservation du parc} Présentation du parc

Situé au sud de la Côte d'Ivoire, en plein cœur d'Abidjan, le Parc National du Banco a été créé par le décret du 31-10-1953 avec une superficie de 3.474 ha. Ce massif forestier fut classé réserve forestière en 1926 (LACHENAUD O., 2006). Selon le découpage administratif actuel, le parc est à cheval sur les communes d'Adjamé, de Yopougon et d'Abobo. Il est géré par la Direction de la Zone Sud de l'OIPR(2002). Il est à 5²1'et $5^{\circ} 25^{\prime}$ de Latitude Nord ; $4^{\circ} 1^{\prime}$ et $4^{\circ} 5^{\prime}$ de Longitude Ouest (Figure 1 ). Le parc 
national du Banco abrite une relique de forêt dense sempervirente, psammohygrophile (Lauginie et al. 1996). La rivière Banco, qui prend sa source dans le nord du parc et le traverse jusqu'au sud, constitue l'élément essentiel du réseau hydrographique. Il est considéré comme le poumon de la ville d'Abidjan. Cet écosystème forestier fait partie du réseau de Parcs Nationaux et Réserves naturelles qu’on dénomme aire protégée, gérée en Côte d'Ivoire par l'Office Ivoirien des Parcs et Réserves.

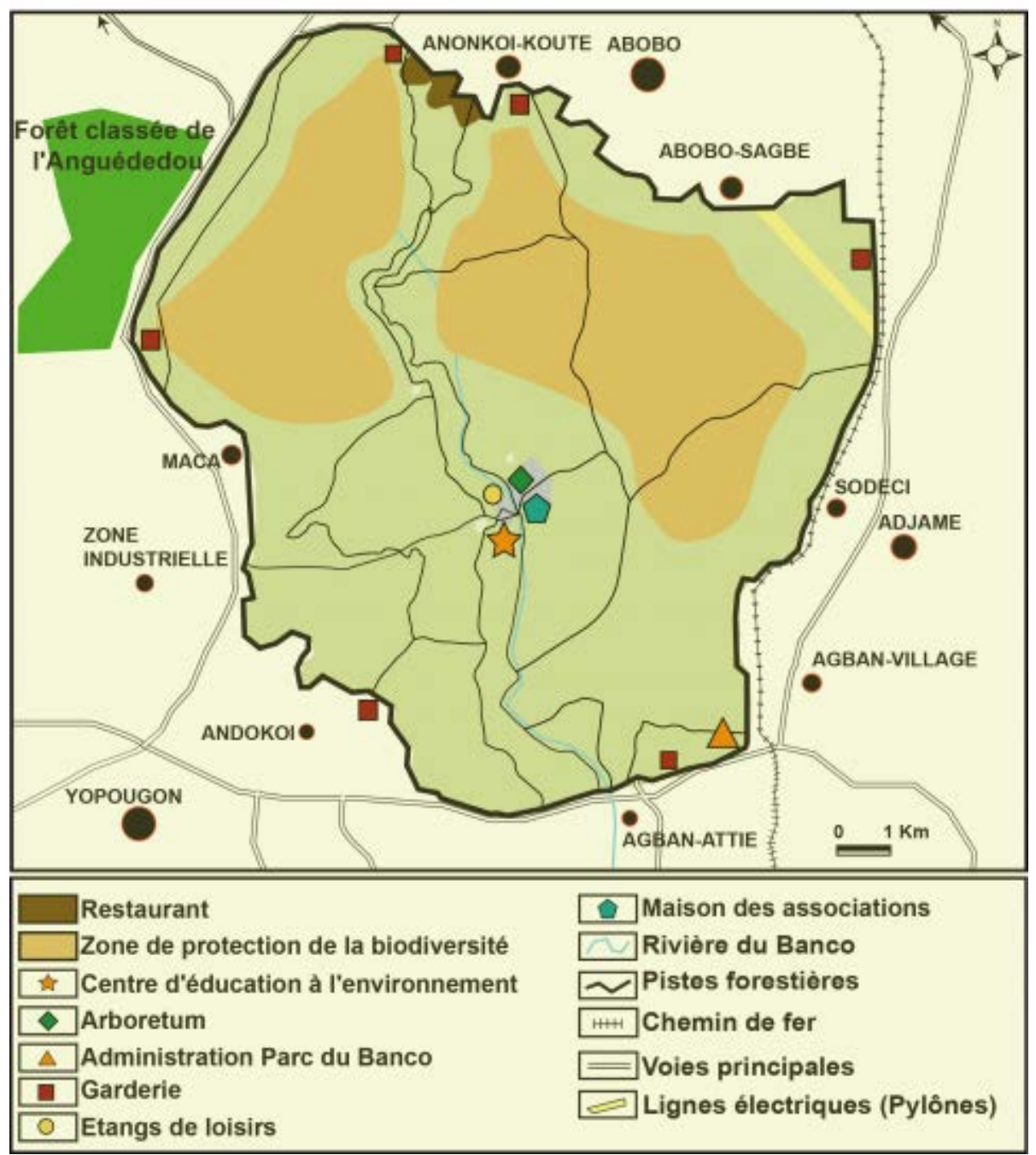

Source : Carte d’aménagement du parc national du Banco éditée par l’Office Ivoirien des Parcs et Réserves (OIPR, 2004).

Figure 1 : Carte présentant les zones périphériques du parc national du Banco

\section{De la conservation dirigiste à l'approche participative}

Deux grandes approches s'observent en matière de protection et de conservation des aires protégées. Il s'agit de la conservation dirigiste et de la 
conservation participative. Toutes les aires protégées de Côte d'Ivoire, tout comme celles de l'Afrique francophone et anglophone, ont connu dans un premier temps la période de la conservation dirigiste et font actuellement l'expérience de la conservation participative.

\section{La période de la conservation dirigiste}

Les politiques étatiques de gestion des ressources naturelles lancées à partir de la fin du $19^{\text {eme }}$ siècle se sont d'abord inscrites dans un système de relation de domination. Autrement dit, la conservation se faisait au détriment des populations locales. Cette approche dirigiste a provoqué un profond sentiment d'injustice chez les populations locales. On note des relations conflictuelles entre populations locales et conservateurs, notamment l'Etat, l'acteur principal. Durant la période coloniale et même après cette période, les politiques de conservation des Parcs Nationaux et Réserves ont été souvent très répressives vis-à-vis des populations locales. François CONSTATIN (1998), pense que la dégradation et le sabotage des ressources naturelles par les populations sont l'expression d'un désintéressement profond pour les politiques officielles qui, imposées de l'extérieur, tant par les gouvernements que par « l'évidence écologique » occidentales (ROSSI, 1997), méprisent totalement les préoccupations locales. Au parc national du Banco, les populations locales soutiennent avoir été exclues de la gestion du parc depuis sa conservation. Selon la chefferie d'Agban-Village, un des villages autochtones à la périphérie du parc, elle est sollicitée que lors des manifestations officielles au niveau du patrimoine. Les populations durant la période de la conservation dirigiste au niveau du Parc National du Banco se sentaient exclues de toutes activités visant à la conservation du parc. L'exclusion des populations, selon IBO (2005) est la base des échecs des différentes politiques de conservation. S. Koné vivant à la périphérie du parc dit: «je ne connais pas l'importance de ce patrimoine » et M. Samassi d'ajouter : « nous cherchons des espaces pour construire et les gens viennent en monopoliser sans exploiter ». Les populations pensent ne pas être associées dès la création du parc à sa gestion.

A sa création, le Parc National du Banco a été exempté, le long de ses 30 km, d'une Zone Tampon, périmètre susceptible de sécuriser la Zone de Protection contre toutes formes de pressions humaines et d'agressions éventuelles dues à des facteurs socio-culturels notamment (les risques d'inhumation, par exemple).

Cette Zone Tampon devrait recevoir des activités humaines. Ce qui devait maintenir les populations locales proche de l'aire protégée. L’absence d'une telle zone au niveau du PNB montre la gestion unilatérale du patrimoine par les conservateurs. Cette exception n'est pas sans impacts majeurs sur la conservation de cet écosystème et de sa biodiversité. En effet, 
les zones d'habitation sont mitoyennes au parc, ce qui reste un facteur prédisposant à toutes infiltrations au parc. L'exclusion de l'époque contemporaine est non seulement contre-productive mais socialement avilissante puisqu'elle crée des frustrations dont l'exacerbation entraine des destructions des parties du parc pour y bâtir des habitations et autres infrastructures. IBO (2005, Op. cit.) conclura que l'exclusion des populations des politiques publiques de gestion des aires protégées est une réalité. Pour lui, la solution toute trouvée pour faire participer les populations est la méthode participative.

\section{La période de la gestion participative du Parc National du Banco}

La notion de participation, envisage la conservation de la nature par la gestion du social. Il faut intégrer les populations locales dans les stratégies de conservation par une approche participative basée sur l'éducation et le renforcement des capacités de ces populations (RODARY, 2003). La conservation s'intègre donc dans l'univers social des acteurs locaux. Dans cette dynamique de gestion participative, les populations bénéficieront de projet de développement. Compagnon (2000) pense que l'engagement de la banque Mondiale dans les programmes de conservation et l'afflux de financements internationaux sont les éléments qui ont encouragé la mise en place de projets communautaires, aussi appelés conservation participation ou encore projet de conservation et de développement intégrés (PCDI). Le Congrès International des Parcs de 1982 (Barrow et Fabricus, 2002) adopte un nouveau modèle de conservation, une «community-friendly conservation ». Cette stratégie repose essentiellement sur un renforcement de l'aide aux communautés locales, par l'éducation, le partage des revenus issus de la conservation, la mise en place de projets de développement dans les zones périphériques des aires protégées.

La gestion participative du Parc National du Banco incombe à l'Office Ivoirien des Parcs et Réserves (OIPR) de manière générale et en particulier à la direction zone sud de l'OIPR. Dans le cadre de cette gestion, il est mis en place un comité de gestion locale comprenant plusieurs acteurs ayant des compétences diverses. Selon les résultats de nos enquêtes de terrain, 70 personnes, soit $67 \%$ de nos enquêtés affirment ne pas être informés de l'existence du comité de gestion. Par contre, 33\% des enquêtés soutiennent connaitre son existence. En effet, K. Yapi, habitant de la zone d’Andokoi relate : « j’ai entendu parler du comité de gestion locale, mais j'ignore son rôle.». Cette position est appuyée par Sanogo, un riverain d’Agban-Attié, qui affirme : « le comité de gestion locale n’a aucune activité visible sur le terrain. D’ailleurs, ce comité ignore la plupart du temps, les réalités que vivent les populations locales concernées ». . Les populations sont insérées dans le comité local de gestion du parc mais, elles y sont qu’à 
titre figuratif. La majorité des populations riveraines du parc soutiennent ne pas être associées à la gestion de ce site de récréation et ne jamais avoir bénéficié de fonds et autres intérêts provenant de la gestion du dit site.

En ce qui concerne les projets de développement, ils sont quasiinexistants à la périphérie au dire des populations autochtones des villages d'Andokoi et d'Agban situé respectivement dans les communes de Yopougon et d'Adjamé.

Pour Rodary (1998), la participation repose sur un idéal démocratique. Les populations sont impliquées dans les projets, on note qu’il y a une réduction du rôle de l'Etat et un transfert des compétences vers la périphérie. Si les populations locales n’adaptent pas leurs pratiques en fonction de ce qui est défini comme durable, ou « écologiquement correct » au niveau mondial, elles sont exclues du processus de développent dont elles font l'objet. La participation aboutit à l'inclusion sociale de ceux dont les pratiques entrent dans le système mondial et à l'exclusion de ceux dont les pratiques n’entrent pas dans ce système. ». La gestion participative des aires protégées n’a toujours pas permis de trouver une solution aux conflits récurrents entre conservateurs et populations locales d'une part et à la dégradation des patrimoines d'autre part. Au niveau du Parc National du Banco, on note une dégradation accélérée du patrimoine bien qu'on ait mis en place le comité de Gestion local. Jacques Weber (1998), soutient que la résolution des conflits et des problèmes de dégradation passe par une «vraie participation » qu'il qualifie de «médiation patrimoniale ». En effet pour Jacques Weber, la «médiation patrimoniale » ou encore " médiation environnementale » est une alternative à la « participation ». Parce qu'elle permet d'associer plus durablement « conservation » et « développement ». Il soutient aussi que : «La 'médiation patrimoniale” part de l'obtention d’un agrément sur le long terme pour revenir du futur vers le présent. Elle repose sur l'hypothèse que le long terme n'était pas prévisible. Il est encore décidable ; et que prévoir, c’est gouverner ».

\section{IMPACT DES ACTIONS ANTHROPIQUES SUR LE PARC Le parc et ses zones périphériques}

L’environnement du parc national du Banco connaît de profondes transformations depuis les décennies 1970. Ces mutations vertigineuses sont liées à plusieurs facteurs dont l'urbanisation rapide du district d'Abidjan, de la croissance démographique et économique et industrielle. Depuis 1955, la forêt dense du parc couvrait une superficie estimée à 5462 ha (33,44\%) tandis que la forêt secondaire occupe une surface de 9220 ha (56,4\%) (MECV, 1995). A cette époque, existaient déjà des traces urbaines disparates. Mais les seules traces d'anthropisation visibles étaient celles de l'école forestière et de campements agricoles au centre et au sud-ouest du 
parc. Le principal centre urbain qui jouxte le parc national du Banco était situé au sud-est dans la commune d’Adjamé. Néanmoins, quelques villages et nouveaux quartiers font leur apparition dans le nord et le sud-ouest du parc. En effet, selon Hauhouot (2002), dans les années 1950, Abidjan est une ville moyenne, faiblement peuplée (55000 habitants) avec de nombreux villages situés à sa périphérie. En 1960, à l’indépendance de la Côte d’Ivoire, Abidjan en est devenu la capitale politique et connaît une évolution importante de sa population et de ses infrastructures. De 500000 habitants en 1970, Abidjan comptait près de 5000000 d'habitants en 2006 (INS, 2006).

Cette croissance urbaine rapide a provoqué la destruction des écosystèmes forestiers qui ont été remplacés par des habitations à la périphérie du parc. En outre, la construction des infrastructures et équipements urbains liés à l'aménagement et à l'extension de la ville d'Abidjan ont exacerbé la situation. Ces aménagements ont provoqué un isolement de la forêt du parc national du Banco dans la métropole abidjanaise.

En 1998, le parc national du Banco et sa périphérie sont couverts de 2826 ha de forêt dense humide et 624 ha de forêt secondaire (Gerard .B ; Nakouma.S et al, 2014). L'ensemble de ces types de végétation forestière avec une strate ligneuse représente au total $68 \%$ de la zone. Si à première vue, le parc national du Banco, dans ses limites administratives est quasiment couvert de forêt, les zones urbaines autour de sa périphérie représentent $32 \%$ de la zone. L'extension de la métropole abidjanaise consomme les espaces forestiers périphériques, c'est le cas du PNB sur le front occidental de l'agglomération d'Abidjan.

En 1998, environ les trois quarts (3/4) de la forêt sont encerclés par les surfaces bâties de l'agglomération, notamment dans le sud, l'ouest, l'est et le nord du parc national du Banco. Cette extension des espaces urbains densément peuplés et insuffisamment aménagés est à l'origine d'une dégradation vertigineuse de la forêt. Au nord-est du parc national Banco, des sols nus occupés par les pylônes électriques isolent une partie de la forêt.

\section{Les actions anthropiques}

Les populations vivant autour du Parc National du Banco mènent plusieurs activités détruisant ainsi les zones protégées du parc. Cette exploitation illégale des ressources forestières est liée aux types de localité (villages, quartiers résidentiels et précaires...) à la périphérie du Parc National du Banco. En effet, les observations que nous avons réalisées au cours de notre enquête ont permis de voir l'ampleur de ces exploitations illicites et des pollutions liquides et solides qui menacent le parc (Hauhouo, 2002). La nature et l'intensité des activités dominantes varient en fonction 
des localités périphériques. Le parc national du Banco est bordé au nord et au sud par des anciens villages des ethnies «Ebrié et Attié » situés, suite aux différentes extensions spatiales, dans l'aire urbaine du district d'Abidjan. Ces villages sont majoritairement habités par des autochtones qui exploitent quotidiennement le parc et revendiquent une grande partie du territoire du parc national du Banco. Ces revendications foncières influencent donc les rapports de ces populations avec le parc qu'elles considèrent comme leur territoire traditionnel. En effet, les activités agricoles et les pratiques foncières spéculatives sont encore visibles et dominantes chez les autochtones se réclamant propriétaires réels du parc. La situation de leurs villages dans le district d’Abidjan reste identique malgré les nombreuses activités économiques urbaines que bénéficient la ville d’Abidjan depuis des décennies. Elles ont peu d'influence sur les pratiques agricoles et les rapports à la terre de ces villageois. Les populations de ces villages ne bénéficiant pas du tout des retombées du District de la ville d'Abidjan et se croyant surtout isolés et en marge du développement de leur zone, se vengent en exploitant les portions du parc. À l'opposé de ces villages, le parc est également bordé de quartiers résidentiels, majoritairement habités par des marchands qui tiennent des petits commerces dans les différentes communes d'Abidjan. Les populations de ces quartiers pratiquent des activités de service et s’intéressent peu aux ressources du parc. Le risque d'exploitation directe de la forêt par cette catégorie de la population est donc moindre par rapport aux populations des villages. Au-delà de ces localités indiquées, notre enquête a relevé aussi la présence de nouveaux quartiers qui se situent au nord-ouest du parc. Les résidents de ces quartiers appartiennent essentiellement à la classe moyenne notamment des travailleurs du secteur public ou privé ayant acheté des maisons à proximité du parc à la faveur d’opérations immobilières. L'enquête a donc permis d'identifier et de les classer en fonction de leurs impacts potentiels sur l'exploitation forestière illégale dans le parc.

Les zones où les revendications foncières sont les plus importantes se situent au nord-est et au sud du parc. Ces zones contestées se localisent à proximité des villages riverains d’Anonkoua Kouté et de Sagbé au nord, Agban-attié et Agban-village au sud-est, et Andokoi au sud-ouest. Ces zones sont intensément exploitées par les villageois notamment pour y prélever les bois de feu, les plantes traditionnelles et médicinales. Les pressions foncières au nord-est du parc sont les plus intenses. Les autochtones revendiquent ouvertement la portion de forêt qui a été isolée suite au passage des lignes électriques de haute tension à l'intérieur du parc. Cette portion du parc, séparée du reste de la forêt, a même été l’objet de nombreuses tentatives de lotissement de la part des populations autochtones. Ces tentatives ont souvent provoqué des heurts violents entre les forces de police forestière et les villageois. Les riverains dans cette partie du parc y sont hostiles. Pour 
eux, la présence de ce massif forestier est synonyme d’insécurité. En effet, ces populations pensent que la forêt est un refuge de bandits armés, de malfaiteurs et d'esprit malsain.

Les limites administratives jouent également un rôle important dans les comportements des populations riveraines de la forêt du Banco. Les espaces forestiers situés à proximité des limites administratives matérialisées par un mur, une barrière ou un panneau sont moins « agressés » que ceux en contact direct avec les populations. L’infiltration des limites administratives varie également selon les zones frontalières et les pratiques des populations locales. Les données de l'enquête autour du parc national du Banco montrent que les zones les plus vulnérables sont situées au sud-ouest, à l'est et au nord-est du parc. Ces espaces exploités font partie des zones où les limites administratives de la forêt ne sont pas matérialisées. Les infiltrations dans cette partie du parc sont plus importantes. Par contre à l'extrême sud du parc, les limites administratives matérialisées par l’autoroute font que les forêts de cette partie du parc sont moins affectées par les infiltrations illégales. La présence de cette voie routière, au trafic dense, met partiellement cette partie du parc à l'abri des infiltrations illégales des populations riveraines. Enfin, les zones situées au nord-ouest du parc national du Banco, à la lisière de l'aire protégée de l’Anguédédou, sont faiblement affectées par les activités humaines. La protection naturelle du parc par la forêt voisine de l'Anguédédou et la faible densité des populations dans cette zone entraîne une faible exploitation des ressources forestières situées à proximité de cette limite.

\section{Recommandations pour gestion durable du parc national du banco Le développement de l'écotourisme et des projets communautaires autour du parc}

Actuellement, les autorités du parc mènent un travail important de communication et de sensibilisation pour stimuler le tourisme local qui avait connu son âge d’or dans les années 1960. Le parc présente une variété de sites touristiques qui offre sa biodiversité et ses attraits: flore et faune typique de la forêt dense humide tropicale ; sentiers de découverte du milieu naturel ; jardins botaniques ; source de la rivière Banco et piscine naturelle au nord du parc. Le parc national du Banco dispose également d'infrastructures, notamment un restaurant-bar situé à proximité de l'arboretum, une maison forestière, une école forestière ouverte en 1938.

Le tourisme au Banco se trouve donc au cœur de la stratégie de valorisation économique rationnelle de la faune et de la flore du Banco. Les recettes tirés de l'écotourisme pourraient participer, selon les administrateurs du parc, à financer d'une part sa protection, et d'autre part à améliorer les 
conditions de vie des riverains pour ainsi les détourner des pratiques prédatrices.

Aussi, les aménagements entrepris autour du parc rentrent dans cet objectif d'un développement touristique florissant de cette aire protégée. En 2002, grâce à un financement du Fonds National de l'Environnement (FNDE), des initiatives ont été entreprise avec la réhabilité de certains services en état de ruine notamment : garderies, la piscine et les étangs piscicoles.

En outre, pour assurer le développement touristique, plusieurs activités ont été menées pour mettre en place des attractions et des structures d'accueil : formation de guides d'accueil et organisation de visites guidées dans le parc à l'attention des écoles ; création d'un musée de la forêt et création à la périphérie du parc de boutiques de souvenirs, l’aménagement de l'arboretum ainsi que le reprofilage des pistes forestières et des sentiers touristiques rendant ainsi les visites plus agréables. Les populations, jeunes comme les femmes participent aux micro-projets à caractère environnemental, notamment par l'attribution aux riverains de concessions liées à l'exploitation du parc, des activités sportives (course de vélos), transport et gestion des déchets, etc.

\section{La sensibilisation, un outil efficace d'information et de communication}

Parmi les stratégies de sensibilisation et en raison de la diversité des formes d'organisation sociale des populations autour du parc, nous suggérons une valorisation de la fonction de chefs de communauté ethnique riverains c’est-à-dire les populations Ebrié et Attié vivant autour du parc. Dans la situation de fragilité qui caractérise actuellement, le parc, l'anticipation rationnelle des risques et des incertitudes engendre un repli des populations sur leurs communautés ethniques d'appartenance. Face à la menace qui pèse sur le parc national du Banco, il $\mathrm{y}$ a une tendance à envisager des stratégies collectives de résistance, mais autour de leurs chefs. Sur des thématiques de sensibilisation comme la lutte contre les feux de brousse (zone prison-civile), la divagation des troupeaux (zone N'dotré), lutte contre le braconnage et l'exploitation des espèces végétales etc., il faut plutôt envisager de susciter plus d'adhésion spontanée collective et individuelle. Celles-ci permettent d’éviter le plus possible le recours à la force. Pour ce faire, il faudra :

- approcher tous les chefs des communautés ethniques (Ebrié, Attié et les Autres communautés...) présentes autour du parc pour leur notifier la revalorisation de leur fonction dans la nouvelle stratégie de la gestion de ce parc,

- les sensibiliser sur le rôle qu'on voudrait les voir jouer auprès de leurs administrés et les infractions sur lesquelles il doit attirer leur attention, 
- suggérer à chaque chef de communauté de proposer des sanctions applicables par lui et ses notables et par type d'infraction. La procédure pourrait être la suivante : sommation à deux reprises du contrevenant, réunir toute la communauté et la prendre à témoin, l'appel aux forces des Eaux et Forêts.

Une fois que cela est fait, une grande manifestation au cours de laquelle sera remis des "'Titres de responsabilité individuels"' portant leur nom et leur photo. Ces titres font fonction d'attestation de reconnaissance de la légitimité de ces chefs pour leur engagement pour la protection durable du parc.

\section{Conclusion}

Cette étude a permis de décrire les stratégies de conservation du Parc National du Banco avec ses deux périodes que sont la conservation dirigiste et la gestion participative. Les résultats des enquêtes de terrain ont montré que le Parc National du Banco, durant ces deux temps de la conservation a continué d’être dégradé et infiltré. L’implication des populations à la gestion du parc reste mitigée et les conflits entre gestionnaires et populations sont toujours récurrents. Cette ambigüité a entrainé la perte de nombreuses ressources floristiques et fauniques d'une part et la perte de certaine partie du parc au profit de certains quartiers périphériques d'autre part. Le parc national du banco situé en plein cœur de la ville d'Abidjan est considéré comme son «poumon ». Avec le réchauffement climatique ce patrimoine doit être sauvegardé en vue d'une réduction de la température qui ces dernières années ne fait que monter. Pour que ce patrimoine demeure le « poumon » de la ville d'Abidjan sa gestion doit incomber à tous. Une vraie gestion participative doit être appliquée dans le sens que souhaite Jacques Weber. Il s’agit de la médiation patrimoniale.

\section{References:}

1. Akindes, F., (1997), Étude diagnostic pour la mise en place d'un dispositif participatif autour des parcs nationaux. Coordination du PCGAP, Abidjan, 88 p.

2. Barima, Y.S.S., (2007), Dynamique du paysage d'une zone de transition forêt-savane dans le département de Tanda à l'Est de la Côte d'Ivoire. Mémoire DEA, Université libre de Bruxelles, Belgique, 65 p.

3. Barrow, E. Fabricius, C. (2002). Do rural people really benefit from protected areas management? Rhétoric or reality? “parks”, 12, 2, pp 67-79. UICN, Gland. In: Les aires protégées en Afrique. Conservation et développement: quelle intégration? Montamat Helene (2007) Collection «Clio en Afrique » n²2- printemps - été 
4. Bigot, S., (2004), Variabilité climatique, interactions et modifications environnementales - l'exemple de la Côte d’Ivoire. Mémoire d'habilitation de direction à la recherche, Université de Lille, Lille, 398 p.

5. Brou, Y.T., (2005), Climat, mutations socio-économiques et paysages en Côte d'Ivoire, HDR, Université de Cocody/ Université des Sciences et Technologies de Lille, 212 p.

6. Chatelain, C., (1996), Possibilités d'application de l'imagerie satellitaire à haute résolution pour l'étude des transformations de la végétation en Côte d'Ivoire forestière. Thèse de doctorat, Université de Genève, $177 \mathrm{p}$.

7. Compagnon, D. (2000). Impératifs et contraintes de la gestion communautaire, in :.Administrer l'environnement en Afrique : gestion communautaire, conservation et développement durable. Compagnon, D. et Constantin, F. (2000). Paris, Karthala, pp 13-35

8. Constantin, F. (1998). « Nihil novi sub sole ». Pas de raccourci vers l'efficience écologique : de la domination hégémonique et élististe à la participation populaire. Communication personnelle au colloque panafricain sur la gestion communautaire des ressources naturelles et le développement durable, Harare, Zimbabwe (24-27 Juin) 1996.

9. Dibi N'da, H. (2007). Étude et suivi par télédétection et Système d'Informations Géographiques d'une aire protégée soumise aux pressions anthropiques : Cas du Parc National de la Marahoué, Thèse de doctorat unique, Université de Cocody, UFR des Sciences de la Terre et des Ressources Minières, 140 p.

10. Duchesne, V., (2002), Des lieux sacrés aux sites classés. Évolution du contrôle des ressources naturelles dans le Sud-Est ivoirien. In Patrimonialiser la nature tropicale. Dynamiques locales, enjeux internationaux. Cormier-Salem M.-C., Juhé-Beaulaton D., Boutrais J., Roussel, B., Paris, IRD, collection "Colloques et séminaires" p. 419-438.

11. DPN, (2003), Rôle des parcs nationaux et réserves analogues et problématiques de conservation en Côte d'Ivoire. Ministère de l'Environnement, Abidjan, Côte d'Ivoire, pp. 19-21

12. Ibo, J. (2005). Le journal des sciences sociales: les politiques publiques de gestion des réserves naturelles : réalité de l'exclusion des populations locales et de l'approche participative. Revue scientifique du GIDIS $n^{\circ} 2$.

13. Lauginie, F., (1996), Propositions pour l'avenir des parcs nationaux et réserves naturelles de Côte d'Ivoire. DDC/MINAGRA/WWF, Abidjan, 86 p. 
14. Sako, N ; Beltrando, G ; Atta, K, L ; N'da, D, H ; et Brou, T. (2013). «Dynamique forestière et pression urbaine dans le Parc national du Banco (Abidjan, Côte d'Ivoire) » in ,VertigO - la revue électronique en sciences de l'environnement [En ligne], Volume 13 Numéro 2 | septembre 2013, mis en ligne le 06 octobre 2013, consulté le 18 septembre 2016. URL : http://vertigo.revues.org/14127 ; DOI : 10.4000/vertigo.14127

15. Oszwald, J., (2005), Dynamique des formations agroforestières en Côte d'Ivoire (des années 1980 aux années 2000). Suivi par télédétection et développement d'une méthode cartographique. Thèse de doctorat, Université de Lille 1, Lille, 302 p.

16. Rodary, E. (1998). De la conservation participative à la promotion de l'économie libérale. Les politiques de conservation de la faune en Zambie et au Zimbabwe. Politique Africaine, n 72, pp. 113-129.

17. Rodary, E. (2003). La conservation intégrée en Afrique australe: opportunité ou restriction au développement ? In : Conservation de la nature et développement: l'intégration impossible? Rodary, E. Castellanet, C. Rossi, G Paris: GRET, Karthala, pp 121-141.

18. Rossi, G. (1997). Voulons-nous la participation ? Essai critique sur l'environnement et le développement, In : Regards interdisciplinaires sur les politiques de développement. BARRE, J-F (dir), l'Harmattan, Paris. 